OPEN ACCESS

Edited by:

Bernt Johan von Scholten,

Novo Nordisk, Denmark

Reviewed by:

Muath Alanbaei,

Kuwait University, Kuwait

Asimina Mitrakou-Fanariotou,

National and Kapodistrian University of

Athens, Greece

*Correspondence: Sophie I. Mavrogeni soma13@otenet.gr

Specialty section:

This article was submitted to

Clinical Diabetes,

a section of the journal

Frontiers in Endocrinology

Received: 25 February 2021

Accepted: 12 May 2021

Published: 14 June 2021

Citation:

Mavrogeni SI, Bacopoulou F,

Markousis-Mavrogenis G,

Giannakopoulou A, Kariki O, Vartela V,

Kolovou G, Charmandari E and

Chrousos G (2021) Cardiovascular

Magnetic Resonance

as Pathophysiologic Tool

in Diabetes Mellitus.

Front. Endocrinol. 12:672302.

doi: 10.3389/fendo.2021.672302

\section{Cardiovascular Magnetic Resonance as Pathophysiologic Tool in Diabetes Mellitus}

\author{
Sophie I. Mavrogeni ${ }^{1,2 *}$, Flora Bacopoulou ${ }^{2}$, George Markousis-Mavrogenis ${ }^{1}$, \\ Aikaterini Giannakopoulou ${ }^{3}$, Ourania Kariki ${ }^{1}$, Vasiliki Vartela ${ }^{1}$, Genovefa Kolovou ${ }^{1}$, \\ Evangelia Charmandari ${ }^{4,5}$ and George Chrousos ${ }^{2,5}$
}

\begin{abstract}
${ }^{1}$ Department of Cardiology, Onassis Cardiac Surgery Center, Kallithea, Greece, ${ }^{2}$ University Research Institute of Maternal and Child Health and Precision Medicine, and UNESCO Chair on Adolescent Health Care, National and Kapodistrian University of Athens, Aghia Sophia Children's Hospital, Athens, Greece, ${ }^{3}$ Department of Pediatric Cardiology, Aghia Sophia Children's Hospital, Athens, Greece, ${ }^{4}$ Division of Endocrinology, Metabolism and Diabetes, First Department of Pediatrics, School of Medicine, National and Kapodistrian University of Athens, Aghia Sophia Children's Hospital, Athens, Greece, ${ }^{5}$ Division of Endocrinology and Metabolism, Center for Clinical, Experimental Surgery and Translational Research, Biomedical Research Foundation of the Academy of Athens, Athens, Greece
\end{abstract}

Diabetes mellitus can independently contribute to cardiovascular disease and represents a severe risk factor for premature development of cardiovascular disease. A three-fold higher mortality than the general population has been observed in type 1 diabetes mellitus whereas a two- to four-fold increased probability to develop cardiovascular disease has been observed in type 2 diabetes mellitus. Cardiovascular magnetic resonance, a nonradiative modality, is superior to all other modalities in detecting myocardial infarction. The main cardiovascular magnetic resonance sequences used include a) balanced steadystate free precession (bSSFP) for function evaluation; b) T2-W for oedema detection; c) T1 $W$ for ischemia detection during adenosine stress; and d) late gadolinium enhanced T1-W images (LGE), evaluated 15 min after injection of paramagnetic contrast agent gadolinium, which permit the diagnosis of replacement fibrosis, which appears white in the middle of suppressed, nulled myocardium. Although LGE is the technique of choice for diagnosis of replacement fibrosis, it cannot assess diffuse myocardial fibrosis. The application of T1 mapping (native or pre contrast and post contrast) allows identification of diffuse myocardial fibrosis, which is not detectable my other means. Native T1 and Contrastenhanced T1 mapping are involved in the extracellular volume fraction (ECV) calculation. Recently, $1 \mathrm{H}$-cardiovascular magnetic resonance spectroscopy has been applied to calculate the amount of myocardial triglycerides, but at the moment it is not part of the routine assessment of diabetes mellitus. The multifaceted nature of cardiovascular magnetic resonance has the great potential of concurrent evaluation of function and myocardial ischemia/fibrosis in the same examination and represents an indispensable tool for accurate diagnosis of cardiovascular disease in diabetes mellitus.

Keywords: cardiovascular, magnetic resonance, coronary artery disease, heart failure, myocardial fibrosis, diabetes, cardiac MRI, cardiac function 


\section{INTRODUCTION}

The clinical implications of diabetes mellitus (DM) on the cardiovascular system are profound with serious consequences that are reflected in patients' survival worldwide. Insulin resistance can directly contribute to cardiovascular disease (CVD) (1). Furthermore, asymptomatic patients with DM demonstrate impaired myocardial perfusion rate index (MPRI), compared with normal volunteers (2). Cardiovascular magnetic resonance (CMR) can detect occult myocardial lesions and reduced microvascular perfusion in patients with early type 2 DM (T2DM) (3).

Patients with DM have increased CVD morbidity/mortality as a result of various pathophysiologic changes, including epicardial coronary artery disease (CAD) leading to myocardial infarction (MI), microvascular CAD, endothelial dysfunction, cardiac remodeling due to diffuse myocardial fibrosis, fatty myocardial infiltration and diastolic dysfunction leading to heart failure (HF). Finally, peripheral vascular disease, involving carotid arteries and brain vasculature, may cause claudication and stroke, respectively (4).

\section{CARDIOVASCULAR DISEASE IN DM}

Three-fold higher mortality has been identified in patients with type 1 diabetes mellitus (T1DM) compared to controls, due to premature atherosclerosis in both men and women (5). In this population, CVD events appear a decade earlier, in comparison with nondiabetic subjects (6). A recent meta-analysis found that the CVD mortality rate was 5.7 for men and 11.3 for women with $\operatorname{T1DM}(7,8)$. Recent findings showed a 10 -fold increased risk of CVD mortality in T1DM depending on glycemic levels (9). Furthermore, coronary artery calcifications were identified in $>70 \%$ of men and $>50 \%$ of women over 45 years with T1DM (10). Adequate management of CVD risk factors led to a $29 \%$ reduction in the cardiac death risk over next 10 years (11). This resulted to a CVD risk of 2.3 in men and 3.0 in women (12). The excess risk in women with T1DM cannot be attributed to the usual CVD risk factors (13) and supports that women with T1DM are not protected against CVD (14). It is important to notice that male and female patients with T1DM have the same risk of CVD (15).

CAD and myocardial infarction (MI) are two to four times more common in patients with T2DM (16). Therefore, T2DM is an independent factor for stroke and CVD (17), with almost 70\% of T2DM patients dying at age < 65 years due to CVD (16). T2DM patients without CAD are at the same risk with patients with previous MI (18). Classic CVD risk factors can additionally augment the CVD risk in T2DM. It is well documented that insulin resistance and hyperglycemia lead to low-grade inflammation triggering endothelial dysfunction (19-21). Blood inflammatory indices together with increased platelets activity are the most important causative factors $(22,23)$.

\section{NON-INVASIVE CARDIOVASCULAR IMAGING IN DM}

Various noninvasive imaging modalities have been applied to diagnose early cardiovascular involvement in DM. Among them, echocardiography is the cornerstone modality applied in clinical practice, because it can be used for bedside evaluation, has low cost, is radiation free, and is widely available. However, echocardiography has some serious limitations, including dependency of operator and acoustic window, low reproducibility, and lack to provide information about tissue characteristics. Additionally, it is unable to differentiate between epicardial and microvascular CAD and cannot detect small MIs that do not cause significant wall motion abnormalities (4). Furthermore, exercise echocardiography, using dobutamine as a pharmacologic stress factor, is often suboptimal in T2DM, due obesity, which usually coexists with DM (4).

SPECT imaging is the most commonly used modality to assess myocardial ischemia in DM. However, in DM patients, SPECT has some serious limitations. Its spatial resolution of $\approx 7$ $\mathrm{mm} \times 9 \mathrm{~mm}$ does not allow detection of subendocardial ischemia. SPECT relies on regional differences in myocardial blood flow and, therefore, is less accurate in patients with triple vessel disease and balanced ischemia, diffuse microvascular dysfunction and non-transmural MI (4). Furthermore, obese patients usually have lower signal in the inferolateral wall that leads to false-positive results. Despite these limitations, a systematic analysis of SPECT studies showed that in DM patients with and without symptoms, a normal SPECT indicates an annual event rate for $\mathrm{MI} /$ cardiac death around $1.9 \%$ (24). Finally, SPECT uses radioactive tracers that are not indicated in the young DM patients with rapid disease progression and atypical presentations, who need repeated reevaluation scans (4).

Computed coronary angiography (CTA) can play a role in assessing DM patients, and there is a great debate regarding its use in asymptomatic patients and the value of calcium screening as a gatekeeper for further ischemia testing (25). However, data from the FACTOR 64 study showed that in DM patients with or without symptoms, CTA does not improve their management or outcome (26).

\section{WHICH IS THE PLACE OF CMR IN DM EVALUATION?}

Cardiovascular Magnetic Resonance is a non-radiative modality, with high spatial resolution $(0.3-1 \mathrm{~mm})$ capable to provide details regarding both function and tissue characteristics in the same study (27). CMR is superior to all other modalities in detecting MI (4). The ICELAND MI study revealed an incidence of $27 \%$ $\mathrm{MI}$ in the general population between 67 and 93 years and $32 \%$ in patients with DM (27). Importantly, the number of unrecognized MIs was high in both groups (17\% and $21 \%)$, demonstrating 
significant underestimation of MI in DM patients. It should be noticed that unrecognized MIs had the same negative impact on patients' prognosis with known MIs, despite their smaller size. Similarly, other investigators found evidence of MI in $28 \%$ of $\mathrm{DM}$ patients, and this was the best prognostic factor of future adverse events (28). Turkbey et al. studied 1,017 patients with T1DM (741 using Gd contrast agent, approximately 49 years, DM duration equal to 22 years) and found increased left ventricular (LV) mass with reduced end-diastolic volumes. These parameters were related to CVD risk factors, HbA1c and macroalbuminuria (29). In $4.3 \%$ of DM patients, an MI was found by CMR in contrast to only $1.4 \%$ by clinical evaluation. Additionally, Rijzewijk et al. found a strong correlation between myocardial steatosis and diastolic abnormalities in DM patients (30). Finally, Heydari et al. evaluated the utility of CMR first-pass perfusion imaging using vasodilatory stress with adenosine or regadenoson for risk classification of DM patients. After examination of 173 symptomatic patients with DM using CMR, and follow-up over $2.9 \pm 2.5$ years, they found that inducible myocardial ischemia, defined as at least one positive segment of more than one voxel thickness lasting for at least three heartbeats, was the strongest predictor of outcome. Patients with DM with neither ischemia nor MI had a $0.5 \%$ event rate for cardiac death or MI, those with no inducible ischemia $1.4 \%$ and those with inducible ischemia $8.2 \%$ per year. We should also emphasize that the DM duration did not correlate with the prevalence of ischemia (31).

\section{CMR TECHNIQUES TO ASSESS CARDIOVASCULAR DISEASE IN DIABETES MELLITUS}

Cardiovascular sequelae constitute a substantial burden on the treatment of DM at both the patient and population levels (32). CMR can provide non-invasive, highly reproducible, radiationfree information about the status of all cardiac tissues (7). The basic pulse sequences to succeed this target include (33):

1. Balanced steady-state free precession (bSSFP). bSSFP is increasingly important to assess myocardial function. It is also the ideal approach to evaluate cardiac anatomy/mass/wall motion/biventricular/atrial function and cardiac remodeling (Figure 1) (34).

2. T2- weighted images (T2-W). These images are produced due to increased water amount taken place during oedema $(35,36)$. Oedema is the acute reaction of myocardium to any kind of damage either ischemic (myocardial infarction) or inflammatory (any type of myocarditis). It may be localized or diffuse, subendocardial, or transmural following the territory of coronary arteries as in CAD (Figure 2) and subepicardial or intramyocardial as in myocarditis (Figure 3).

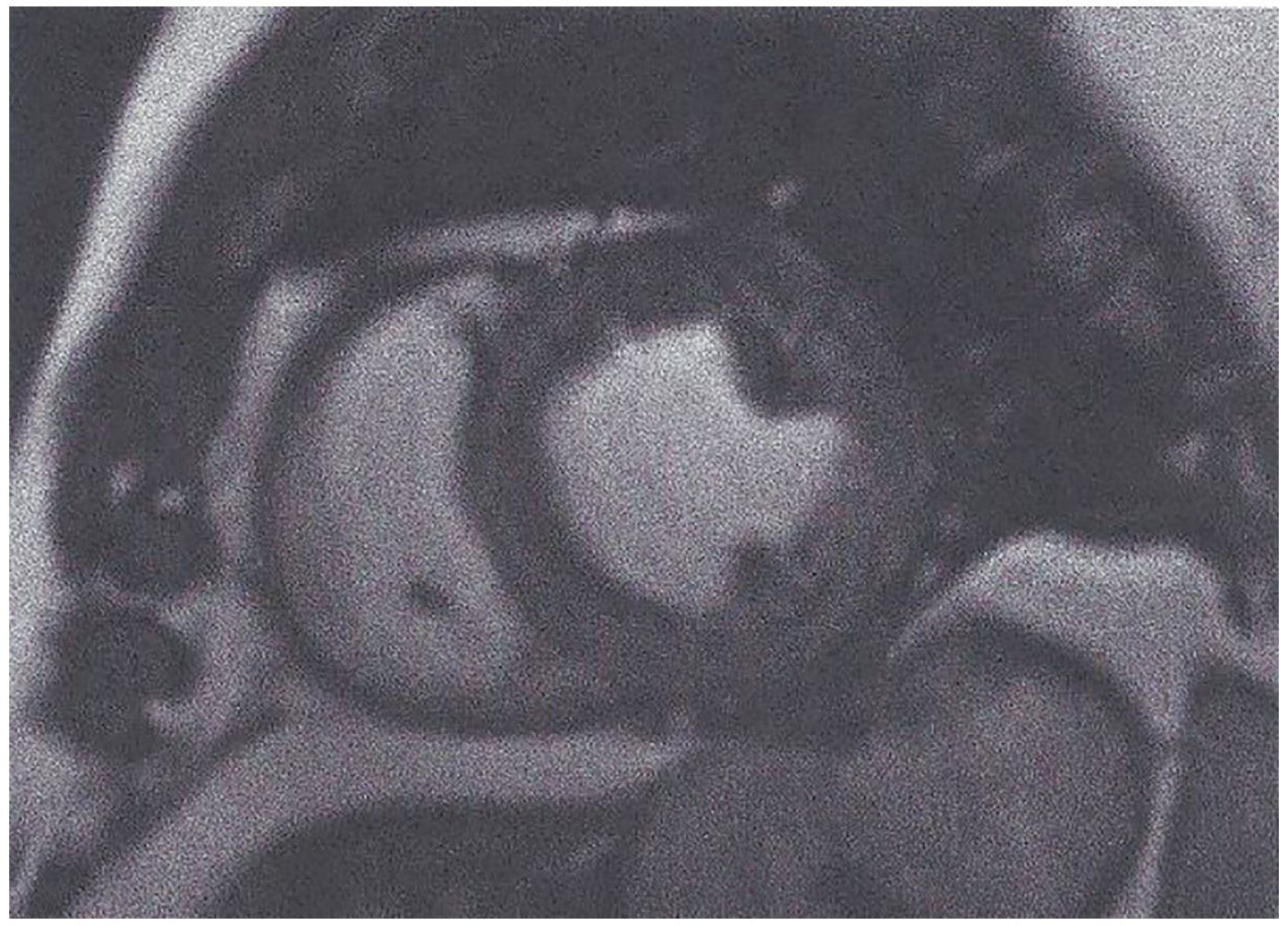

FIGURE 1 | Short axis SSFP image used for myocardial function assessment. This figure is original and based on data from Mavrogeni et al. 


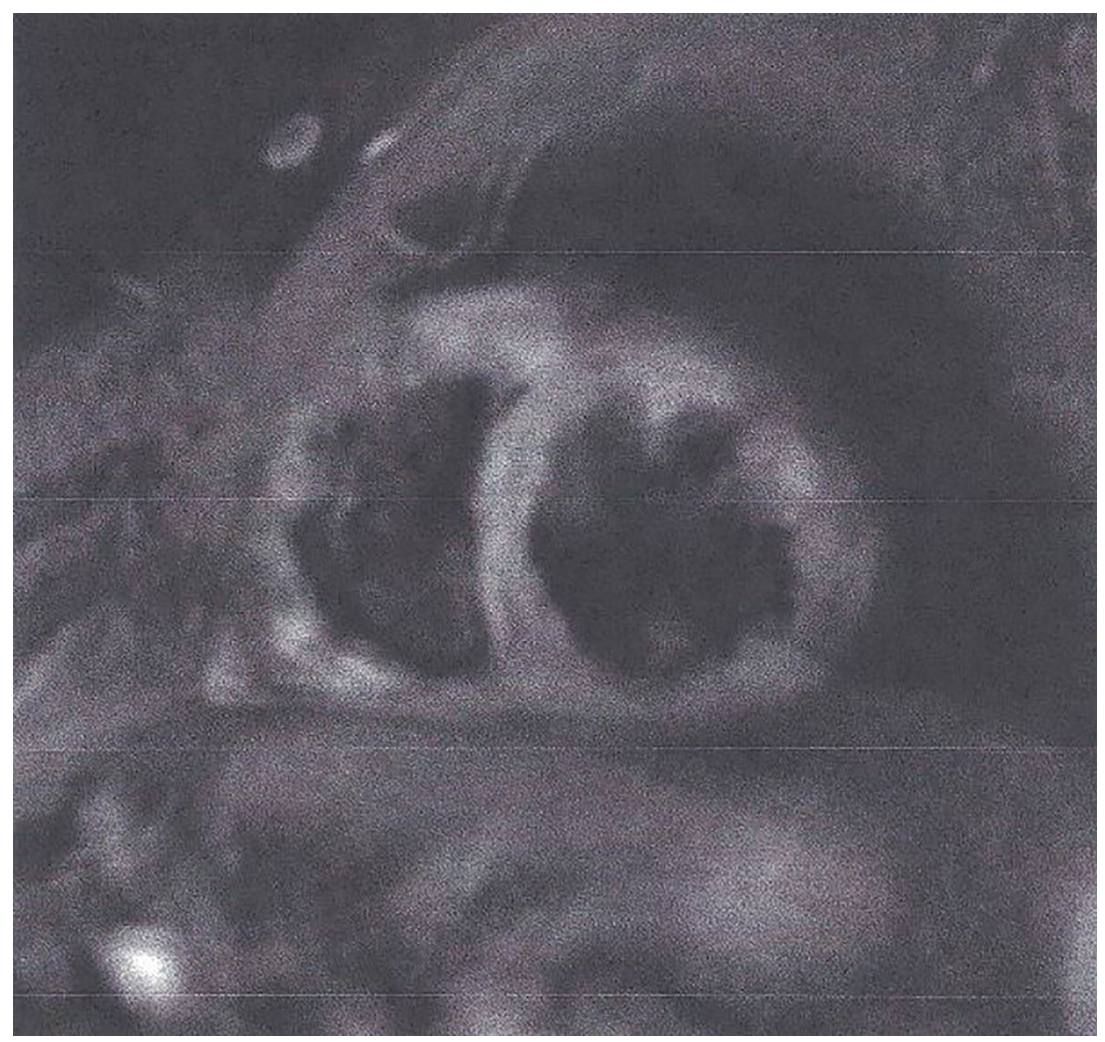

FIGURE 2 | STIR T2 image showing transmural oedema in myocardial infarction. This figure is original and based on data from Mavrogeni et al.

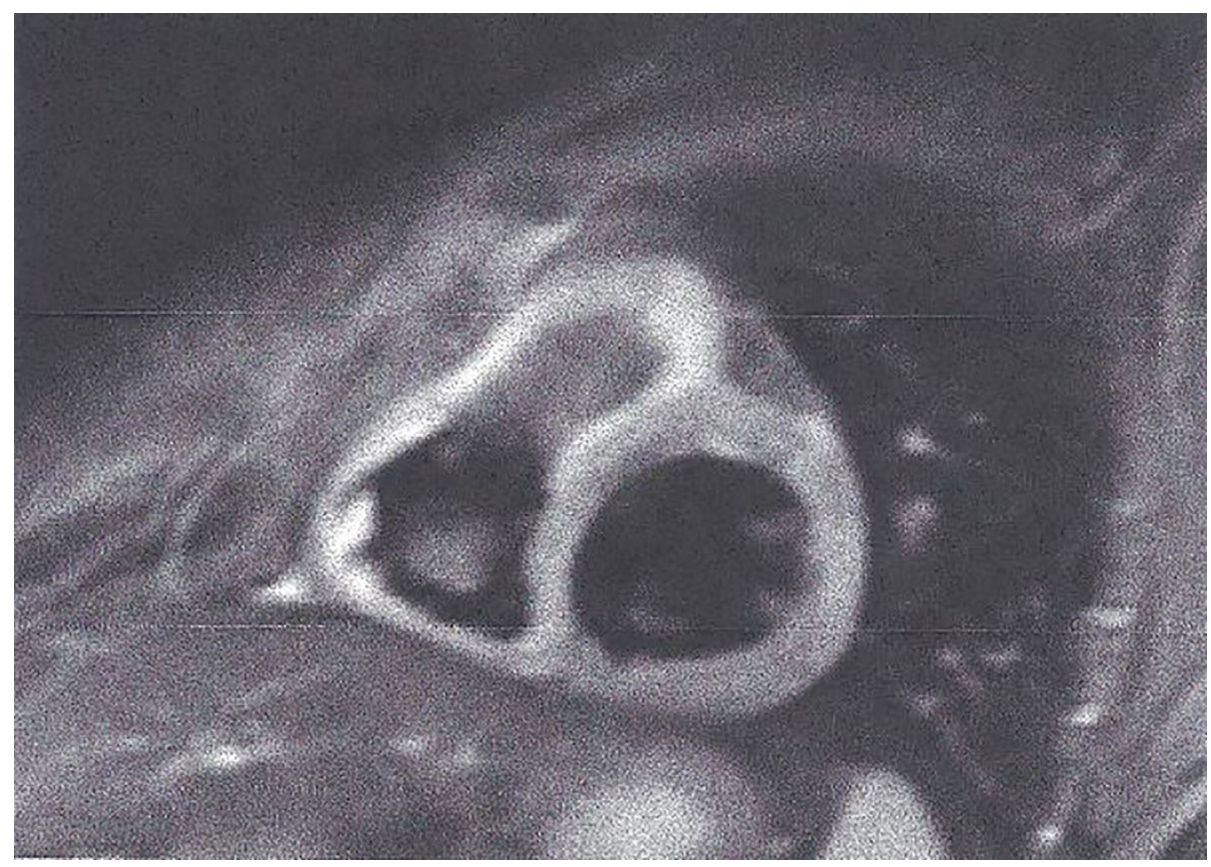

FIGURE 3 | STIR T2 image showing subepicardial oedema in myocarditis. This figure is original and based on data from Mavrogeni et al. 
Oedema presents high intensity on short tau inversion recovery (STIR) images. However, these images may present various limitations (37), including low contrast between healthy/edematous areas, dependency on field homogeneity, blurring due to cardiac motion, subendocardial slow flow hyperintensity, and subjective differences in visual interpretation $(37,38)$.

To overcome the STIR limitations, T2 mapping has been developed, based on the transverse relaxation time of each voxel. At $1.5 \mathrm{~T}$, the normal myocardium $\mathrm{T} 2$ value was measured at $52 \pm 3 \mathrm{~ms}$ by Giri et al. in 14 healthy volunteers and at $55 \pm 5 \mathrm{~ms}$ in 73 healthy volunteers by Wassmuth et al. and were related to body surface area (BSA) or heart rhythm and had excellent reproducibility $(39,40)$.

3. T1-weighted images (T1-W). The T1 relaxation time, an index of how quickly the spins return to equilibrium after a radiofrequency (RF) pulse, is a main parameter of tissue contrast (41). T1-W without contrast agent is ideal for anatomy evaluation. Late gadolinium enhanced T1-W images (LGE), assessed $15 \mathrm{~min}$ after gadolinium, permit the detection of myocardial fibrotic areas, which appear as bright in a nulled, black myocardium "bright is dead" (Figure 4) (32). According to the type and location of LGE, it is attributed to $\mathrm{CAD}$, if the lesion follows the distribution of coronary arteries. In contrast, subepicardial or patchy LGE usually in the inferolateral wall is characteristic of any kind of myocarditis. This technique performs equally well in the diagnosis of both acute and chronic myocardial scar (32).

T1-W after pharmacologic stress using adenosine or regadenoson and bolus injection of paramagnetic contrast agent can provide accurate, reproducible information about myocardial perfusion during stress (Figure 5) (41). This approach has independent prognostic utility and can reclassified CVD risk in DM patients, referred for ischemic assessment (31). Furthermore, almost $1 / 4$ of asymptomatic DM patients with Framingham risk $\geq 20 \%$ had occult CAD and reduced global MPRI, compared with normal subjects (42).

4. Parametric imaging (T1, T2 imaging, ECV). Although LGE is well validated as the technique of choice for the detection of replacement fibrosis, it has serious limitations in the assessment of diffuse myocardial fibrosis, because it is based on the signal intensity differences between fibrotic and normal myocardium to provide image contrast. To overcome this problem, a CMR imaging technique called T1 mapping has been created. T1 mapping identifies early myocardial fibrosis, which is undetectable using the current blood biomarkers (43). Normal values of T1 mapping in $1.5 \mathrm{~T}$ are $995.8 \pm 30.9 \mathrm{~ms}$ and $1,183.8 \pm 37.5 \mathrm{~ms}$ at $3 \mathrm{~T}(44)$ (Figure 6).

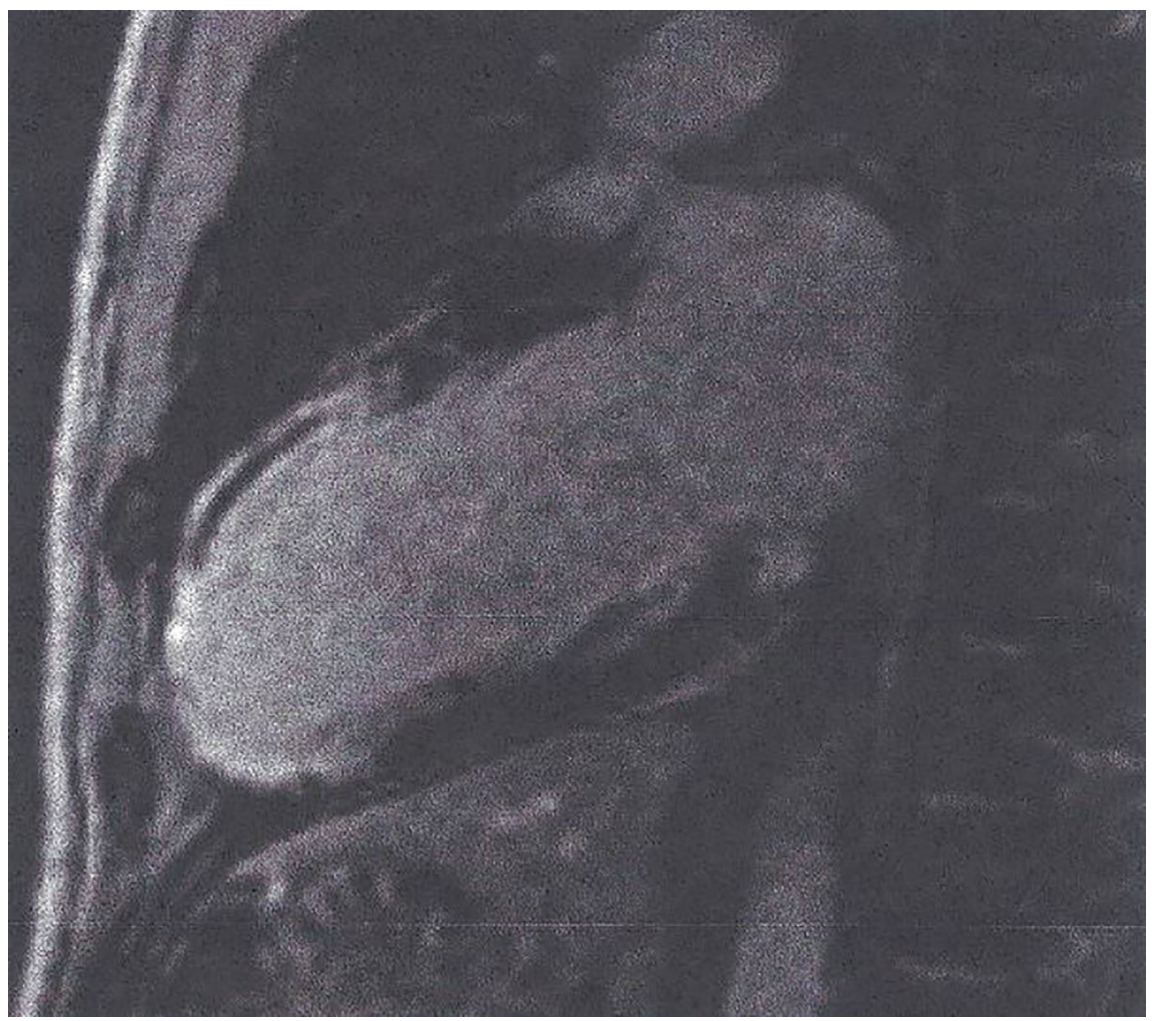

FIGURE 4 | LGE image showing extensive myocardial infarction in anterior wall (bright area) with microvascular obstruction (dark area within the white area of scar) in a patient with T2DM. This figure is original and based on data from Mavrogeni et al. 


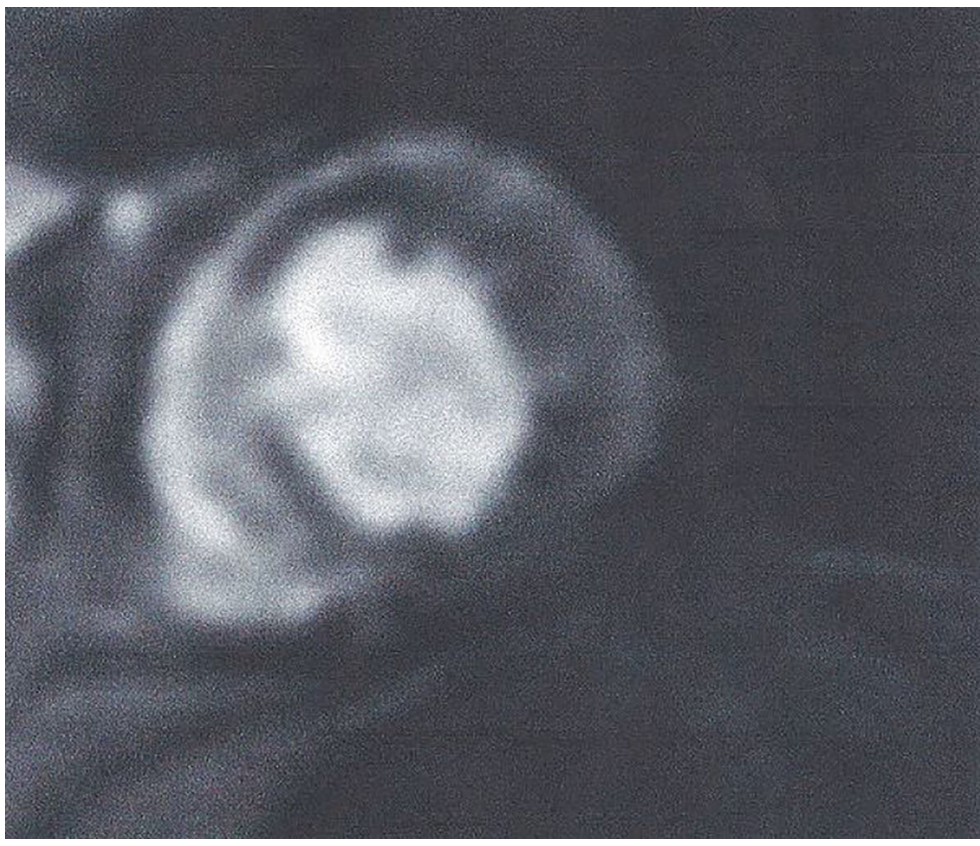

FIGURE 5 | Adenosine stress CMR image showing extensive perfusion defect in anterior and inferolateral wall of LV (dark area) in a patient with T2DM. This figure is original and based on data from Mavrogeni et al.

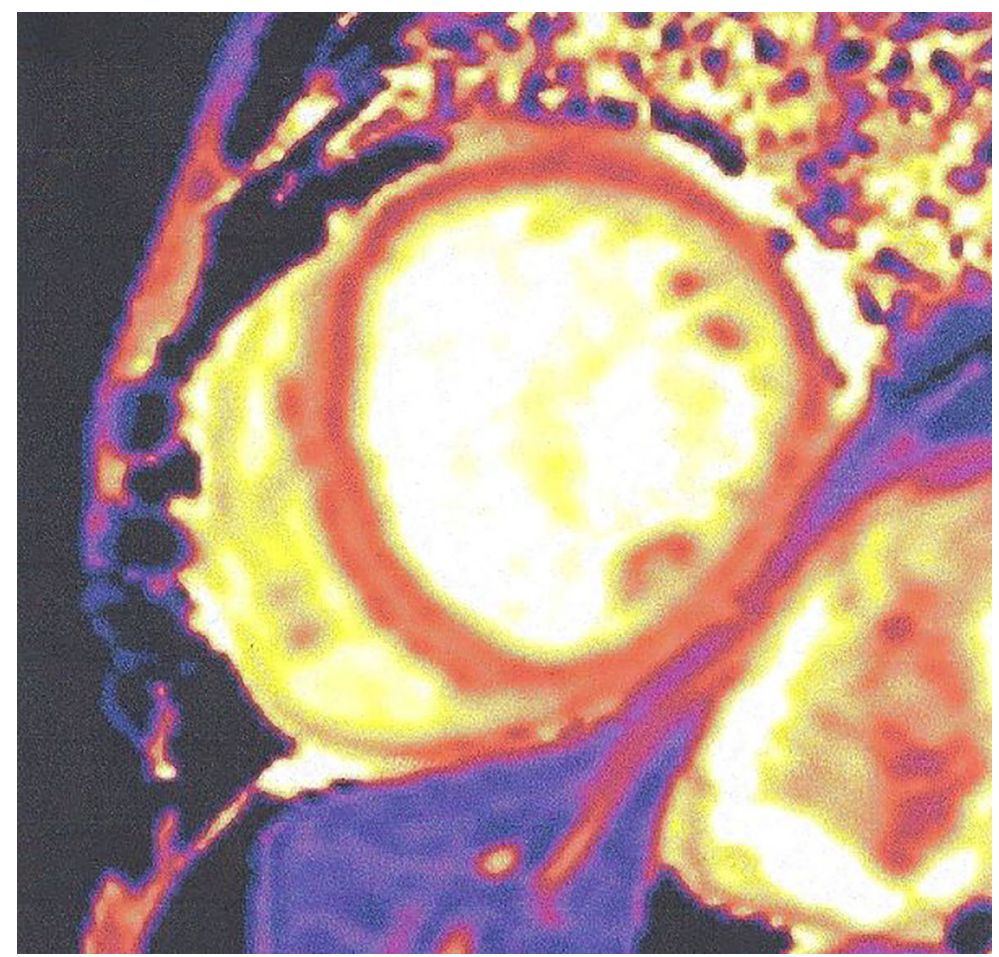

FIGURE 6 | Increased native T1 mapping in a patient with diabetic cardiomyopathy. This figure is original and based on data from Mavrogeni et al. 
- Contrast-enhanced T1 mapping is used for the extracellular volume fraction (ECV) calculation together with native T1 mapping. The estimation of the ECV is an index of inter-and extra-cellular area and measurement of myocardial/blood T1 before/after gadolinium together with hematocrit is needed, with hematocrit representing the cellular fraction of blood.

- Normal ECV values equal to $25.3 \pm 3.5 \%$ were found in healthy subjects, examined at $1.5 \mathrm{~T}$ scanners (45). With the exception of amyloidosis, an increased ECV is the result of collagen presence, as in case of systemic sclerosis and other cardiomyopathies (46). Low ECV was found in thrombus and lipomatous metaplasia and can be calculated either from T1 mapping images or directly visualized on ECV maps images (46).

Compared to native $\mathrm{T} 1$ relaxation times, contrast-enhanced $\mathrm{T} 1$ values are depended on various parameters, such as contrast dose, time between contrast injection, and T1 imaging and renal function and therefore are more variable. ECV, on the other hand, is a physiologic index derived from the ratio of T1 values. Therefore, ECV is a more reproducible index in different magnetic fields, acquisition techniques, and vendors than both native/post-contrast T1 (46). Furthermore, ECV has excellent correlation with histologic assessment of the collagen than T1 mapping (47).

Using this approach, it was proven that elevated ECV is an independent factor of mortality in DM representing a novel non-invasive biomarker to evaluate the severity of diabetic heart disease (48). Additionally, DM was found to have increased T1, T2 mapping and ECV, compared to controls, independently of LGE (49). Furthermore, native T1 mapping is sensitive to myocardial infarcts and scarring (16) and allows the follow-up of longitudinal changes during treatment trials (16). Recently, adenosine stress and rest $\mathrm{T} 1$ mapping detected ocult abnormalities of the microvascular circulation, without the use of gadolinium, providing diagnostic information for early therapeutic intervention (50). Finally, patients with prediabetes or DM and preserved LVEF had increased LV remodeling, suggesting early alterations during the disease process (51). The CMR techniques used for myocardial evaluation of DM are presented in Table $\mathbf{1 .}$

\section{ADVANCED CMR TECHNIQUES USED IN DM}

CMR found a correlation between myocardial lipid accumulation and LV diastolic dysfunction, independently of age, blood pressure, and obesity in T2DM (30) and improvement after anti-diabetic treatment $(52,53)$. $\mathrm{Ng}$ et al. described an association between CMR assessed myocardial triglycerides and longitudinal strain measured using echocardiography, suggesting a subclinical effect of lipids on myocardial function in T2DM (54). In another study by Korosoglou et al. evaluated strainencoded CMR, 1H-CMR spectroscopy for triglycerides quantification and adenosine stress-CMR for myocardial perfusion reserve and found that only myocardial triglycerides were correlated with LV diastolic dysfunction in T2DM. This correlation persisted even after adjustment for demographics and clinical parameters (55).

CMR has been also used to evaluate the effect of treatment. A randomized study to pioglitazone, metformin or placebo for 24 weeks in $78 \mathrm{~T} 2 \mathrm{DM}$ men without CVD showed no effect of treatment on myocardial lipids and energetic, measured by $1 \mathrm{H}$ and 31P CMR spectroscopy, respectively (56). Similar findings showed the lack of rosiglitazone effect on myocardial lipids using $1 \mathrm{H}$-CMR spectroscopy (57). It is also important to mention that the prolonged caloric restriction in T2DM decreases the myocardial triglycerides leading to amelioration in LV diastolic function (52). Finally, increased myocardial lipid amount was found in patients with impaired glucose tolerance and normal LVEF (52).

In a study using cardiac phosphocreatine-to-ATP ratio, it was found that this index was lower in T2DM patients compared with controls (58). Furthermore, this parameter was correlated with LV diastolic dysfunction (59). Finally, T2DM evaluation using positron emission tomography (PET) and CMR reported a correlation between hepatic triglycerides, measured using $1 \mathrm{H}$ MRI and impaired cardiac perfusion, function, and high-energy phosphate metabolism (60). Furthermore, 31P-CMR assessment in T1DM has shown that the presence of abnormal cardiac highenergy phosphate metabolism is independent of myocardial perfusion and DM duration (61). The capability of these techniques to evaluate the cellular phosphate metabolism and

TABLE 1 | CMR sequences for myocardial evaluation in patients with DM.

\begin{tabular}{|c|c|c|c|}
\hline Pulse sequence & Myocardial characteristic assessed & Type of study & Cardiac disease in DM \\
\hline Steady-state free precession (SSFP) & $\begin{array}{l}\text { Function of LV-RV } \\
\text { Wall motion/thickness } \\
\text { Myocardial mass }\end{array}$ & Clinical & $\begin{array}{l}\text { LV-RV dysfunction } \\
\text { LV hypertrophy }\end{array}$ \\
\hline Inversion recovery for Late Gadolinium Enhancement (LGE) & $\begin{array}{l}\text { Replacement fibrosis due to Ml } \\
\text { Myocarditis } \\
\text { Infiltrative disease }\end{array}$ & Clinical & $\begin{array}{l}\text { Myocarditis } \\
\text { Myocardial infarction } \\
\text { Infiltrative disease }\end{array}$ \\
\hline First-pass T1 imaging using stress & Myocardial stress perfusion & Clinical & $\begin{array}{l}\text { Myocardial ischemia due to micro-macro- } \\
\text { CAD }\end{array}$ \\
\hline T1 mapping/ECV & Diffuse myocardial fibrosis & Clinical & $\begin{array}{l}\text { Extent and severity of diffuse myocardial } \\
\text { fibrosis }\end{array}$ \\
\hline $1 \mathrm{H}$ and 31P CMR spectroscopy & Cardiac triglycerides and cardiac energetics & Experimental & $\begin{array}{l}\text { Pre-clinical detection of myocardial } \\
\text { involvement }\end{array}$ \\
\hline
\end{tabular}


to quantify the myocardial phosphate may be of crucial significance in the validation of novel treatments in DM. However, these exciting applications at the moment are not available in the clinical practice.

Finally, water/fat separation CMR sequences, as well as ${ }^{1} \mathrm{H}$ MRS, have provided valuable insight into the relationships between epicardial adiposity, myocardial fat content, and obesity-related cardiac disease. Whether increased epicardial adiposity in healthy subjects can lead to myocardial fat loading and subclinical impairment of myocardial function is an issue of great interest, with important clinical implications for obesityrelated cardiac disease. However, further studies are needed to understand whether quantification of epicardial adiposity and myocardial fat content in normal hearts have any diagnostic, prognostic or therapeutic impact to reverse established myocardial disease in humans (62).

\section{CARDIOVASCULAR MAGNETIC RESONANCE: PRO AND CONTRA}

Within the significant advantages of CMR are included the lack of radiation, the great reproducibility of the measurements and the capability of performing tissue characterization, independently of blood biomarkers. Its high spatial resolution is of great value in the accurate measurement of myocardial mass, volumes, and biventricular ejection fraction. Furthermore, it is the most reliable modality for the identification of ischemia, fibrosis, edema, and inflammation. However, important disadvantages include limited access to MRI system, lack of expertise, and increased examination cost not covered by the re-imbursement system in most countries. Additionally, there are limitations, such as claustrophobia, long time of examination/processing, inability to examine patients with non-CMR compatible devices. Furthermore, non-cooperative patients cannot be examined without sedation and in patients with renal failure the use of contrast agents is restricted, due to the risk of nephrogenic fibrosis. However, the high cost of CMR examination maybe counterbalanced by the advantage of timely diagnosis and treatment of CVD in DM. In a recently published systematic review regarding the CVD cost in DM patients, it was shown that from a population level, CVD costs contributed between $20 \%$ and $49 \%$ of the total direct costs of treating T2DM. The median annual costs per patient for CVD, coronary artery disease, heart failure, and stroke were, respectively, $112 \%, 107 \%, 59 \%$, and 322\% higher compared with those for T2DM patients without CVD. On average, treating patients with $\mathrm{CVD}$ and $\mathrm{T} 2 \mathrm{DM}$ resulted in a cost increase ranging from $\$ 3418$ to $\$ 9705$ compared with treating patients with T2DM alone (32). Finally, the EACVI preparatory course to certification in CMR has a cost of 1280 euros for each candidate.

To assess sensitivity/specificity of CMR vs other imaging modalities, the CE-MARC study (63) evaluated 235 women and 393 men with suspected angina using CMR, SPECT and $\mathrm{x}$-ray angiography. In this study, the CMR evaluation, including adenosine stress/rest perfusion, cine imaging, late gadolinium enhancement, and magnetic resonance coronary angiography, found that the sensitivity in women and men was similar $(88.7 \%$ versus $85.6 \%$; $\mathrm{P}=0.57$ ), as was the specificity ( $83.5 \%$ versus $82.8 \%$; $\mathrm{P}=0.86)$. For SPECT, the sensitivity was significantly worse in women than in men $(50.9 \%$ versus $70.8 \%$; $\mathrm{P}=0.007)$, but the specificities were similar ( $84.1 \%$ versus $81.3 \%$; $\mathrm{P}=0.48$ ). The sensitivity in both the female and male groups was significantly higher with CMR than SPECT $(\mathrm{P}<0.0001$ for both), but the specificity was similar $(\mathrm{P}=0.77$ and $\mathrm{P}=1.00$, respectively). For perfusion-only components, CMR outperformed SPECT in women (area under the curve, 0.90 versus $0.67 ; \mathrm{P}<0.0001$ ) and in men (area under the curve, 0.89 versus $0.74 ; \mathrm{P}<0.0001$ ). Diagnostic accuracy was similar in both sexes with perfusion CMR $(\mathrm{P}=1.00)$ but was significantly worse in women with SPECT $(\mathrm{P}<0.0001)(63)$.

In cardiac amyloidosis (CA), LGE-CMR showed a sensitivity of $95 \%$ and a specificity of $98 \%$ for the diagnosis of CA. The combination of a characteristic LGE pattern indicating CA with unremarkable monoclonal protein studies resulted in the diagnosis of ATTR-CA (confirmed by EMB) with a specificity of $98 \%$ [ $95 \%$ confidence interval (CI) $92-100 \%$ ] and a positive predictive value (PPV) of 99\% (95\% CI 92-100\%), respectively, while the EMB risk of complications was $3.13 \%$, but without any detrimental or persistent complications (64).

Finally, a study assessing the diagnostic performance of CMR, compared with endomyocardial biopsy (ENB) in patients with suspected acute myocarditis (AMC) and chronic myocarditis (CMC) showed that the best diagnostic performance was observed in patients with suspected AMC (sensitivity, 81\%; specificity, 71\%; and accuracy, 79\%). In contrast in suspected CMC, CMR was found to be unsatisfactory (sensitivity, 63\%; specificity, 40\%; and accuracy, 52\%) (65).

\section{CMR IN DIABETES: DREAMS AND REALITY}

The 2019 ESC Guidelines on diabetes, pre-diabetes and cardiovascular diseases developed in collaboration with the EASD (66) support that:

1. Resting ECG is recommended in patients with DM with hypertension or suspected CVD

2. Carotid or femoral ultrasound should be considered for plaque detection as $\mathrm{CV}$ risk modifier

3. Screening for CAD with coronary CT angiography and functional imaging may be considered,

4. Coronary artery calcium (CAC) scoring may be considered as risk modifier,

5. Ancle-branchial index (ABI) may be considered as risk modifier,

6. Carotid ultrasound intima-media thickness for CV risk is not recommended. 
It is really impressive that this recent document does not recommend echocardiography, the cornerstone cardiovascular imaging modality, as a screening tool to detect early myocardial changes in DM. However, adolescents with T1D exhibit early changes in blood pressure, peripheral vascular function, and left ventricular myocardial deformation indices with a shift from longitudinal to circumferential shortening (67). Additionally, the evaluation of myocardial mechanics at rest and during dobutamine echo stress showed that T2DM patients presented with altered global diastolic function but preserved systolic function. Furthermore, deformation imaging indexes were similar between groups at rest, but significant differences were noticed under dobutamine infusion for longitudinal strain (68).

It is out of the scope of this article to analyze the role of all recommended imaging modalities in the evaluation of cardiac involvement in DM. However, a glimpse to the literature showed that ECG has low partial sensitivity and specificity for predicting coronary artery stenosis with accuracy ranged $58.5 \%$ to $62.0 \%$ based on coronary artery analysis (69). Furthermore, CT coronary angiography, although it has excellent sensitivity/ specificity, is an ionizing radiation modality and therefore, it cannot be recommended as an annual screening tool (70). Therefore, before expensive, sophisticated modalities will be proposed as screening tools for CAD in DM, we should use the experience coming from the evaluation of other cardiovascular diseases. According to this experience, echocardiography including also the new indices should be incorporated in the annual routine evaluation of DM patients, because it is low cost, easily available, with great expertise between cardiologists and can detect pre-clinical changes in both T1DM and T2DM $(67,68)$. Furthermore, an imaging algorithm including all steps after the echocardiographic evaluation should be proposed (Figure 7). CMR, although it is the ideal imaging modality for CAD detection, it cannot be proposed as a screening tool for all DM patients, due to various reasons mainly including low availability, high expertise, and increased cost. However, there are clear indications for CMR in DM including:

1. Stress CMR perfusion, viability assessment, because it can detect lesions missed by both echocardiography or SPECT;

2. Evaluation of heart failure and arrhythmia in DM patients, because it can reveal the pathophysiologic background behind these entities and guide further treatment;

3. Assessment of ischemia/fibrosis burden in DM patients with known CAD in order to make to final decision regarding revascularization or heart transplantation;

4. Inconclusive echocardiographic evaluation, due to technical reasons, such as obesity or obstructive lung diseases;

5. Doubtful echocardiographic results that need further evaluation, such as differential diagnosis between diabetic cardiomyopathy and heart failure, due to coronary artery disease.

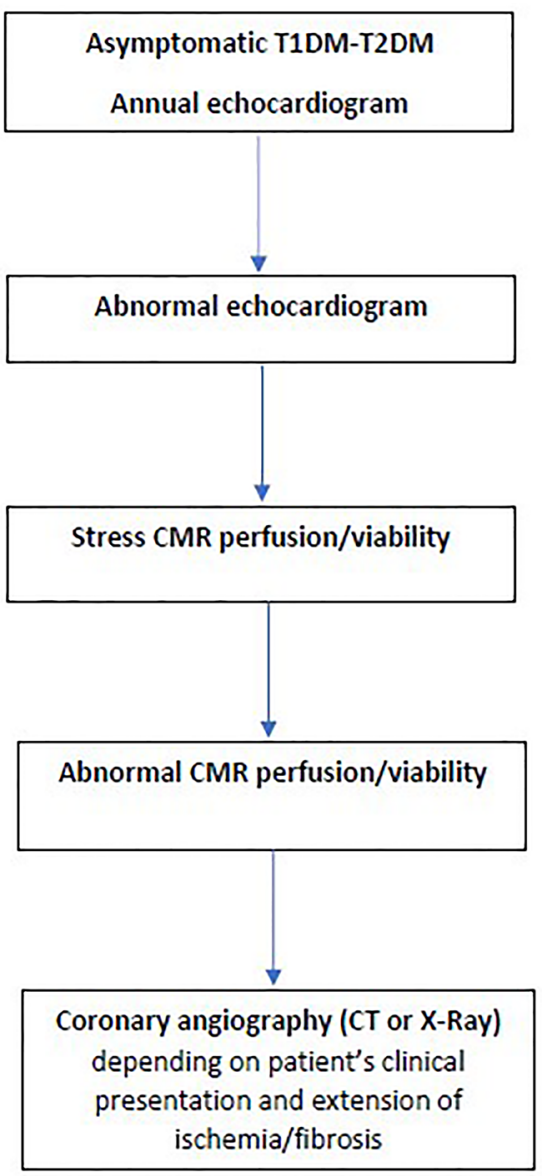

FIGURE 7 | Suggestive algorithm for the evaluation of CAD in DM.

\section{CONCLUSIONS}

The multifaceted nature of CMR allows the assessment of ventricular function/remodeling, myocardial oedema, ischemia during stress, and symptomatic or asymptomatic myocardial replacement/diffuse fibrosis. With increasing availability and easier acquisition/processing, CMR will rapidly become more widely available to the clinicians for early detection of CVD in DM, specifically in subclinical diabetic heart disease.

\section{AUTHOR CONTRIBUTIONS}

$\mathrm{SM}, \mathrm{FB}$, and GM-M contributed to conception and design of the study. SM wrote the first draft of the manuscript. FB, GM-M, AG, OK, VV, GK, EC, and GC wrote sections of the manuscript. All authors contributed to the article and approved the submitted version. 


\section{REFERENCES}

1. Kannel W, Hjortland M, Castelli WC. Role of Diabetes in Congestive Heart Failure: The Framingham Study. Am J Cardiol (1974) 34:29-34. doi: 10.1016/ 0002-9149(74)90089-7

2. de Simone G, Devereux RB, Chinali M, Lee ET, Galloway JM, Barac A, et al. Diabetes and Incident Heart Failure in Hypertensive and Normotensive Participants of the Strong Heart Study. J Hypertens (2010) 28:353-60. doi: 10.1097/HJH.0b013e3283331169

3. Liu X, Yang ZG, Gao Y, Xie LJ, Jiang L, Hu BY, et al. Left Ventricular Subclinical Myocardial Dysfunction in Uncomplicated Type 2 Diabetes Mellitus is Associated With Impaired Myocardial Perfusion: A ContrastEnhanced Cardiovascular Magnetic Resonance Study. Cardiovasc Diabetol (2018) 17:139. doi: 10.1186/s12933-018-0782-0

4. Nagel E. Cardiovascular Magnetic Resonance in Diabetic Patients. The Method of Choice? Circ Cardiovasc Imaging (2016) 9:e004699. doi: 10.1161/CIRCIMAGING.116.004699

5. Khunti K, Davies M, Majeed A, Thorsted BL, Wolden ML, Paul SK. Hypoglycemia and Risk of Cardiovascular Disease and All-Cause Mortality in Insulin-Treated People With Type 1 and Type 2 Diabetes: A Cohort Study. Diabetes Care (2015) 38(2):316-22. doi: 10.2337/dc14-0920

6. Secrest AM, Becker DJ, Kelsey SF, Laporte RE, Orchard TJ. Cause-Specific Mortality Trends in a Large Population-Based Cohort With Long-Standing Childhood-Onset Type 1 Diabetes. Diabetes (2010) 59(12):3216-22. doi: $10.2337 / \mathrm{db} 10-0862$

7. Huxley RR, Peters SA, Mishra GD, Woodward M. Risk of All-Cause Mortality and Vascular Events in Women Versus Men With Type 1 Diabetes: A Systematic Review and Meta-Analysis. Lancet Diabetes Endocrinol (2015) 3 (3):198-206. doi: 10.1016/S2213-8587(14)70248-7

8. Soedamah-Muthu SS, Fuller JH, Mulnier HE, Raleigh VS, Lawrenson RA, Colhoun HM. High Risk of Cardiovascular Disease in Patients With Type 1 Diabetes in the U.K.: A Cohort Study Using the General Practice Research Database. Diabetes Care (2006) 29(4):798-804. doi: 10.2337/diacare. 29.04.06.dc05-1433

9. Lind M, Svensson AM, Kosiborod M, Gudbjörnsdottir S, Pivodic A, Wedel H, et al. Glycemic Control and Excess Mortality in Type 1 Diabetes. N Engl J Med (2014) 371(21):1972-82. doi: 10.1056/NEJMoa1408214

10. Costacou T, Edmundowicz D, Prince C, Conway B, Orchard TJ. Progression of Coronary Artery Calcium in Type 1 Diabetes Mellitus. Am J Cardiol (2007) 100(10):1543-7. doi: 10.1016/j.amjcard.2007.06.050

11. Rawshani A, Rawshani A, Gudbjornsdottir S. Mortality and Cardiovascular Disease in Type 1 and Type 2 Diabetes. N Engl J Med (2017) 377(3):300-1. doi: 10.1056/NEJMoa1608664

12. Livingstone SJ, Looker HC, Hothersall EJ, Wild SH, Lindsay RS, Chalmers J, et al. Risk of Cardiovascular Disease and Total Mortality in Adults With Type 1 Diabetes: Scottish Registry Linkage Study. PloS Med (2012) 9(10):e1001321. doi: 10.1371/journal.pmed.1001321

13. Colhoun HM, Rubens MB, Underwood SR, Fuller JH. The Effect of Type 1 Diabetes Mellitus on the Gender Difference in Coronary Artery Calcification. J Am Coll Cardiol (2000) 36(7):2160-7. doi: 10.1016/S0735-1097(00)00986-4

14. Dabelea D, Kinney G, Snell-Bergeon JK, Hokanson JE, Eckel RH, Ehrlich J, et al. Effect of Type 1 Diabetes on the Gender Difference in Coronary Artery Calcification: A Role for Insulin Resistance? The Coronary Artery Calcification in Type 1 Diabetes (Cacti) Study. Diabetes (2003) 52 (11):2833-9. doi: 10.2337/diabetes.52.11.2833

15. Harjutsalo V, Thomas MC, Forsblom C, Groop PHFinnDiane Study Group. Risk of Coronary Artery Disease and Stroke According to Sex and Presence of Diabetic Nephropathy in Type 1 Diabetes. Diabetes Obes Metab (2018) 20 (12):2759-67. doi: 10.1111/dom.13456

16. Kannel WB, McGee DL. Diabetes and Cardiovascular Disease. JAMA (1979) 241:2035-8. doi: 10.1001/jama.241.19.2035

17. Grundy SM, Benjamin IJ, Burke GL, Chait A, Eckel RH, Howard BV, et al. Diabetes and Cardiovascular Disease: A Statement for Health Professionals From the American Heart Association. Circulation (1999) 100:1134-46. doi: 10.1161/01.CIR.100.10.1134

18. Kim JA, Koh KK, Quon MJ. The Union of Vascular and Metabolic Actions of Insulin in Sickness and in Health. Arterioscler Thromb Vasc Biol (2005) 25 (5):889-91. doi: 10.1161/01.ATV.0000164044.42910.6b
19. Woods M, Mitchell JA, Wood EG, Barker S, Walcot NR, Rees GM, et al. Endothelin-1 is Induced by Cytokines in Human Vascular Smooth Muscle Cells: Evidence for Intracellular Endothelin-Converting Enzyme. Mol Pharmacol (1999) 55:902-9.

20. Petersen KF, Dufour S, Befroy D, Garcia R, Shulman GI. Impaired Mitochondrial Activity in the Insulin-Resistant Offspring of Patients With Type 2 Diabetes. N Engl J Med (2004) 350:664-71. doi: 10.1056/ NEJMoa031314

21. Low Wang CC, Hess CN, Goldfine AB. Clinical Update: Cardiovascular Disease in Diabetes Mellitus: Atherosclerotic Cardiovascular Disease and Heart Failure in Type 2 Diabetes Mellitus - Mechanisms, Management, and Clinical Considerations. Circulation (2016) 133:24. doi: 10.1161/ CIRCULATIONAHA.116.022194

22. Carr ME. Diabetes Mellitus: A Hypercoagulable State. J Diabetes Complications (2001) 15:44-54. doi: 10.1016/S1056-8727(00)00132-X

23. Ross R. Atherosclerosis - an Inflammatory Disease. N Engl J Med (1999) 340:115-26. doi: 10.1056/NEJM199901143400207

24. Acampa W, Cantoni V, Green R, Maio F, Daniele S, Nappi C, et al. Prognostic Value of Normal Stress Myocardial Perfusion Imaging in Diabetic Patients: A Meta-Analysis. J Nucl Cardiol (2014) 21:893-902. doi: 10.1007/s12350-0149918-0

25. Rassi CH, Churchill TW, Tavares CA, Fahel MG, Rassi FP, Uchida AH, et al. Use of Imaging and Clinical Data to Screen for Cardiovascular Disease in Asymptomatic Diabetics. Cardiovasc Diabetol (2016) 15:28. doi: 10.1186/ s12933-016-0334-4

26. Muhlestein JB, Lappé DL, Lima JA, Rosen BD, May HT, Knight S, et al. Effect of Screening for Coronary Artery Disease Using CT Angiography on Mortality and Cardiac Events in High-Risk Patients With Diabetes: The FACTOR-64 Randomized Clinical Trial. JAMA (2014) 312:2234-43. doi: 10.1001/jama.2014.15825

27. Schelbert EB, Cao JJ, Sigurdsson S, Aspelund T, Kellman P, Aletras AH, et al. Prevalence and Prognosis of Unrecognized Myocardial in-Farction Determined by Cardiac Magnetic Resonance in Older Adults. JAMA (2012) 308:890-6. doi: 10.1001/2012.jama.11089

28. Kwong RY, Sattar H, Wu H, Vorobiof G, Gandla V, Steel K, et al. Incidence and Prognostic Implication of Unrecognized Myo-Cardial Scar Characterized by Cardiac Magnetic Resonance in Diabetic Pa-Tients Without Clinical Evidence of Myocardial Infarction. Circulation (2008) 118:1011-20. doi: 10.1161/CIRCULATIONAHA.107.727826

29. Turkbey EB, Backlund JY, Genuth S, Jain A, Miao C, Cleary PA, et al. Dcct/ Edic Research Group. Myocardial Structure, Function, and Scar in Patients With Type 1 Diabetes Mellitus. Circulation (2011) 124:1737-46. doi: 10.1161/ CIRCULATIONAHA.111.022327

30. Rijzewijk LJ, van der Meer RW, Smit JWA, Diamant M, Bax JJ, Hammeret S, et al. Myocardial Steatosis is an Independent Predictor of Diastolic Dysfunction in Type 2 Diabetes Mellitus. J Am Coll Cardiol (2008) 52:1793-9. doi: 10.1016/j.jacc.2008.07.062

31. Heydari B, Juan Y-H, Liu H, Abbasi S, Shah R, Blankstein R, et al. Stress Perfusion Cardiovascular Magnetic Resonance Imaging Effectively Risk Stratifies Diabetic Patients With Suspected Myocardial Ischemia. Circ Cardiovasc Imaging (2016) 9: e004136. doi: 10.1161/CIRCIMAGING.115.004136

32. Einarson TR, Acs A, Ludwig C, Panton UH. Economic Burden of Cardiovascular Disease in Type 2 Diabetes: A Systematic Review. Value Health (2018) 21(7):881-90. doi: 10.1016/j.jval.2017.12.019

33. Mavrogeni SI, Kitas GD, Dimitroulas T, Sfikakis PP, Seo P, Gabriel S, et al. Cardiovascular Magnetic Resonance in Rheumatology: Current Status and Recommendations for Use. Int J Cardiol (2016) 217:135-48. doi: 10.1016/ j.ijcard.2016.04.158

34. Bieri O, Scheffler K. Fundamentals of Balanced Steady State Free Precession MRI. J Magn Reson Imaging (2013) 38(1):2-11. doi: 10.1002/jmri.24163

35. Friedrich MG. Why Edema is a Matter of the Heart. Circ Cardiovasc Imaging (2017) 10(2):e006062. doi: 10.1161/CIRCIMAGING.117.006062

36. Mavrogeni S, Apostolou D, Argyriou P, Velitsista S, Papa L, Efentakis S, et al. T1 and T2 Mapping in Cardiology: "Mapping the Obscure Object of Desire". Cardiology (2017) 138(4):207-17. doi: 10.1159/000478901

37. Eitel I M, Friedrich M. T2-Weighted Cardiovascular Magnetic Resonance in Acute Cardiac Disease. J Cardiovasc Magn Res (2011) 13:13. doi: 10.1186/ 1532-429X-13-13 
38. Wince W, Kim R. Molecular Imaging: T2-weighted CMR of the Area At Riska Risky Business? Nat Rev Cardiol (2010) 7(10):547-9. doi: 10.1038/nrcardio. 2010.124

39. Giri S, Chung YC, Merchant A, Mihai G, Rajagopalan S, Raman SV, et al. T2 Quantification for Improved Detection of Myocardial Edema. J Cardiovasc Magn Res (2009) 11:56-69. doi: 10.1186/1532-429X-11-56

40. Wassmuth R, Prothmann M, Utz W, Dieringer M, Von KnobelsdorffBrenkenhoff F, Greiser A, et al. Variability and Homogeneity of Cardiovascular Magnetic Resonance Myocardial T2-mapping in Volunteers Compared to Patients With Edema. J Cardiovasc Magn Res (2013) 15:27-19. doi: 10.1186/1532-429X-15-27

41. Taylor AJ, Salerno M, Dharmakumar R, Jerosch-Herold M. T1 Mapping: Basic Techniques and Clinical Applications. JACC Cardiovasc Imaging (2016) 9(1):67-81. doi: 10.1016/j.jcmg.2015.11.005

42. Ng MY, Zhou W, Vardhanabhuti V, Lee CH, Tak Yu EY, Fai Wan EY, et al. Cardiac Magnetic Resonance for Asymptomatic Patients With Type 2 Diabetes and Cardiovascular High Risk (CATCH): A Pilot Study. Cardiovasc Diabetol (2020) 19(1):42. doi: 10.1186/s12933-020-01019-2

43. Wynn TA. Cellular and Molecular Mechanisms of Fibrosis. J Pathol (2008) 214(2):199-210. doi: 10.1002/path.2277

44. Granitz M, Motloch LJ, Granitz C, Meissnitzer M, Hitzl W, Hergan K, et al. Comparison of Native Myocardial T1 and T2 mapping At 1.5T and 3T in Healthy Volunteers: Reference Values and Clinical Implications. Wien Klin Wochenschr (2019) 131(7-8):143-55. doi: 10.1007/s00508-018-1411-3

45. Haaf P, Garg P, Messroghli DR, Broadbent DA, Greenwood JP, Plein S. Cardiac T1 Mapping and Extracellular Volume (ECV) in Clinical Practice: A Comprehensive Review. J Cardiovasc Magn Reson (2017) 18:89. doi: 10.1186/ s12968-016-0308-4

46. Moon JC, Messroghli DR, Kellman P, Piechnik SK, Robson MD, Ugander M, et al. Myocardial T1 Mapping and Extracellular Volume Quantification: A Society for Cardiovascular Magnetic Resonance (SCMR) and CMR Working Group of the European Society of Cardiology Consensus Statement. J Cardiovasc Magn Reson (2013) 15:92. doi: 10.1186/1532-429X-15-92

47. Sibley CT, Noureldin RA, Gai N, Nacif MS, Liu S, Turkbey EB, et al. T1 Mapping in Cardiomyopathy At Cardiac MR: Comparison With Endomyocardial Biopsy. Radiology (2012) 265:724-32. doi: 10.1148/ radiol.12112721

48. Khan MA, Yang EY, Nguyen DT, Nabi F, Hinojosa J, Jabel M, et al. Examining the Relationship and Prognostic Implication of Diabetic Status and Extracellular Matrix Expansion by Cardiac Magnetic Resonance. Circ Cardiovasc Imaging (2020) 13(7):e011000. doi: 10.1161/CIRCIMAGING.120.011000

49. Ma J, Xu X, Wang S, Wang R, Yu N. Quantitative Assessment of Early Type 2 Diabetic Cataracts Using T1,T2-mapping Techniques. Br J Radiol (2019) 92 (1103):20181030. doi: 10.1259/bjr.20181030

50. Levelt E, Piechnik SK, Liu A, Wijesurendra RS, Mahmod M, Ariga R, et al. Adenosine Stress CMR T1-Mapping Detects Early Microvascular Dysfunction in Patients With Type 2 Diabetes Mellitus Without Obstructive Coronary Artery Disease. J Cardiovasc Magn Reson (2017) 19(1):81. doi: 10.1186/ s12968-017-0406-y

51. Storz C, Hetterich H, Lorbeer R, Heber SD, Schafnitzel A, Patscheider H, et al. Myocardial Tissue Characterization by Contrast-Enhanced Cardiac Magnetic Resonance Imaging in Subjects With Prediabetes, Diabetes, and Normal Controls With Preserved Ejection Fraction From the General Population. Eur Heart J Cardiovasc Imaging (2018) 19(6):701-70. doi: 10.1093/ehjci/ jex190

52. Hammer S, Snel M, Lamb HJ, Jazet IM, van der Meer RW, Pijl H, et al. Prolonged Caloric Restriction in Obese Patients With Type 2 Diabetes Mellitus Decreases Myocardial Triglyceride Content and Improves Myocardial Function. J Am Coll Cardiol (2008) 52:1006-12. doi: 10.1016/ j.jacc.2008.04.068

53. Zib I, Jacob AN, Lingvay I, Salinas K, McGavock JM, Raskin P, et al. Effect of Pioglitazone Therapy on Myocardial and Hepatic Steatosis in Insulin-Treated Patients With Type 2 Diabetes. J Investig Med (2007) 55:230-6. doi: 10.2310/ 6650.2007 .00003

54. Ng ACT, Delgado V, Bertini M, van der Meer RW, Rijzewijk LJ, Ewe SH, et al. Myocardial Steatosis and Biventricular Strain and Strain Rate Imaging in Patients With Type 2 Diabetes Mellitus. Circulation (2010) 122:2538-44. doi: 10.1161/CIRCULATIONAHA.110.955542
55. Korosoglou G, Humpert PM, Ahrens J, Oikonomou D, Osman NF, Gitsioudis G, et al. Left Ventricular Diastolic Function in Type 2 Diabetes Mellitus is Associated With Myocardial Triglyceride Content But Not With Impaired Myocardial Perfusion Reserve. J Magn Reson Imaging (2012) 35:804-11. doi: 10.1002/jmri.22879

56. van der Meer RW, Rijzewijk LJ, de Jong HWAM, Lamb HJ, Lubberink M, Romijn JA, et al. Pioglitazone Improves Cardiac Function and Alters Myocardial Substrate Metabolism Without Affecting Cardiac Triglyceride Accumulation and High-Energy Phosphate Metabolism in Patients With Well-Controlled Type 2 Diabetes Mellitus. Circulation (2009) 119:2069-77. doi: 10.1161/CIRCULATIONAHA.108.803916

57. McGavock J, Szczepaniak LS, Ayers CR, Abdullah SM, See R, Gore MO, et al. The Effects of Rosiglitazone on Myocardial Triglyceride Content in Patients With Type 2 Diabetes: A Randomised, Placebo-Controlled Trial. Diabetes Vasc Dis Res (2012) 9:131-7. doi: 10.1177/1479164111428628

58. McGavock JM, Lingvay I, Zib I, Tillery T, Salas N, Unger R, et al. Cardiac Steatosis in Diabetes Mellitus: A 1H-Magnetic Resonance Spectroscopy Study. Circulation (2007) 116:1170-5. doi: 10.1161/CIRCULATIONAHA.106.645614

59. Diamant M, Lamb HJ, Groeneveld Y, Endert EL, Smit JWA, Bax JJ, et al. Diastolic Dysfunction is Associated With Altered Myocardial Metabolism in Asymptomatic Normotensive Patients With Well-Controlled Type 2 Diabetes Mellitus. J Am Coll Cardiol (2003) 42:328-35. doi: 10.1016/S0735-1097(03)00625-9

60. Rijzewijk LJ, Jonker JT, van der Meer RW, Lubberink M, de Jong HW, Romijnet JA, et al. Effects of Hepatic Triglyceride Content on Myocardial Metabolism in Type 2 Diabetes. J Am Coll Cardiol (2010) 56:225-33. doi: 10.1016/j.jacc.2010.02.049

61. Shivu GN, Phan TT, Abozguia K, Ahmed I, Wagenmakers A, Henning A, et al. Relationship Between Coronary Microvascular Dysfunction and Cardiac Energetics Impairment in Type 1 Diabetes Mellitus. Circulation (2010) 121:1209-15. doi: 10.1161/CIRCULATIONAHA.109.873273

62. Antonopoulos AS, Antoniades C. Cardiac Magnetic Resonance Imaging of Epicardial and Intramyocardial Adiposity as an Early Sign of Myocardial Disease. Circ Cardiovasc Imaging (2018) 11(8):e008083. doi: 10.1161/ CIRCIMAGING.118.008083

63. Greenwood JP, Motwani M, Maredia N, Brown JM, Everett CC, Nixon J, et al. Comparison of Cardiovascular Magnetic Resonance and Single-Photon Emission Computed Tomography in Women With Suspected Coronary Artery Disease From the Clinical Evaluation of Magnetic Resonance Imaging in Coronary Heart Disease (Ce-MARC) Trial. Circulation (2014) 129(10):1129-38. doi: 10.1161/CIRCULATIONAHA.112.000071

64. Chatzantonis G, Bietenbeck M, Elsanhoury A, Tschöpe C, Pieske B, Tauscher G, et al. Diagnostic Value of Cardiovascular Magnetic Resonance in Comparison to Endomyocardial Biopsy in Cardiac Amyloidosis: A MultiCentre Study. Clin Res Cardiol (2020). doi: 10.1007/s00392-020-01771-1

65. Lurz P, Luecke C, Eitel I, Föhrenbach F, Frank C, Grothoff M, et al. Comprehensive Cardiac Magnetic Resonance Imaging in Patients With Suspected Myocarditis: The Myoracer-Trial. J Am Coll Cardiol (2016) 67 (15):1800-11. doi: 10.1016/j.jacc.2016.02.013

66. Cosentino F, Grant PJ, Aboyans V, Bailey CJ, Ceriello A, Delgado V, et al. 2019 ESC Guidelines on Diabetes, Pre-Diabetes, and Cardiovascular Diseases Developed in Collaboration With the EASD: The Task Force for Diabetes, Pre-Diabetes, and Cardiovascular Diseases of the European Society of Cardiology (ESC) and the European Association for the Study of Diabetes (EASD). Eur Heart J (2020) 41(2):255-323. doi: 10.1093/eurheartj/ehz486

67. Bradley TJ, Slorach C, Mahmud FH, Dunger DB, Deanfield J, Deda L, et al. Early Changes in Cardiovascular Structure and Function in Adolescents With Type 1 Diabetes. Cardiovasc Diabetol (2016) 15:31. doi: 10.1186/s12933-016-0351-3

68. Philouze C, Obert P, Nottin S, Benamor A, Barthez O, Aboukhoudir F. Dobutamine Stress Echocardiography Unmasks Early Left Ventricular Dysfunction in Asymptomatic Patients With Uncomplicated Type 2 Diabetes: A Comprehensive Two-Dimensional Speckle-Tracking Imaging Study. J Am Soc Echocardiogr (2018) 31(5):587-97. doi: 10.1016/j.echo.2017.12.006

69. Mahmoodzadeh S, Moazenzadeh M, Rashidinejad H, Sheikhvatan M. Diagnostic Performance of Electrocardiography in the Assessment of Significant Coronary Artery Disease and its Anatomical Size in Comparison With Coronary Angiography. J Res Med Sci (2011) 16(6):750-5.

70. Meinel FG, Schoepf UJ, Townsend JC, Flowers BA, Geyer LL, Ebersberger U, et al. Diagnostic Yield and Accuracy of Coronary CT Angiography After 
Abnormal Nuclear Myocardial Perfusion Imaging. Sci Rep (2018) 8:9228. doi: $10.1038 / \mathrm{s} 41598-018-27347-8$

Disclaimer: The authors are responsible for the choice and presentation of views contained in this article and for opinions expressed therein, which are not necessarily those of UNESCO and do not commit the Organization.

Conflict of Interest: The authors declare that the research was conducted in the absence of any commercial or financial relationships that could be construed as a potential conflict of interest.
The reviewer AM-F declared a shared affiliation with some of the authors, SM, FB, $\mathrm{EC}$, and GC, to the handling editor at time of review.

Copyright (๑) 2021 Mavrogeni, Bacopoulou, Markousis-Mavrogenis, Giannakopoulou, Kariki, Vartela, Kolovou, Charmandari and Chrousos. This is an open-access article distributed under the terms of the Creative Commons Attribution License (CC BY). The use, distribution or reproduction in other forums is permitted, provided the original author(s) and the copyright owner(s) are credited and that the original publication in this journal is cited, in accordance with accepted academic practice. No use, distribution or reproduction is permitted which does not comply with these terms. 